\title{
Yingkou Port Logistics Information Implementation Status Quo of Research and Development Direction of Research- Yingkou Port Logistics Information Implementation Survey and Development Direction Research \\ Weisheng Zheng ${ }^{1}$ and Yimei Liu $^{2}$
}

\author{
1 Jiangxi Technical College of Manufacturing, Department of Information Engineering; Jiangxi \\ Nanchang; 330095 \\ 2 Jiangxi Technical College of Manufacturing, Department of Information Engineering; JiangXi \\ Nanchang; 330095
}

Keywords: Logistics Information; Port Logistics; EDI

\begin{abstract}
Yingkou Port is one of important ports in Liaoning coastal economic area and also the nearest estuary in Northeast China and eastern Inner Mongolia. It is one of 20 important ports of all coastal areas in China. With the advent of economic globalization and the information age, port logistics information becomes the main development direction for the port, and information is one of the necessary technologies of modern ports, which can facilitate rapid completion of port operations. As hub of land and water transportation, the port serves as both cargo distribution center, as well as the center of information convergence. Timely grasp and use of effective information can effectively manage and improve the port competitiveness, while brings convenience as well as simplifies the business processes. Hence, for giant ports with capabilities over hundred million tons, logistics information will help speed up the inbound and outbound container, and expand throughput capacity.
\end{abstract}

\section{Introduction}

In the past decade, YingKou port has launched information construction as planned in three phases, and achieved good results so far; researchers it can be said that Yingkou Port's logistics information construction and development has had its preliminary effectiveness. In order to accelerate the revitalization of old industrial bases in Northeast China, Yingkou City also proposes the goal of "revitalizing the city with harbor, creating large port and building a modern port city". The transportation, distribution and value-added services of Yingkou Port has an important influence on Yingkou's economic and social development.

The fact that Yingkou Port has become a port with capabilities over hundred million tons, it the result of construction of "Five Points, One Line" coastal economic belt, and also makes it a star in China's port and transportation construction. Port business is the main business of Yingkou Port Group Co., Ltd., and it is particularly necessary to have logistics information for a port with capabilities over hundred million tons. The information technology is throughout the Yingkou Port's major business processes. With the substantial increase in economic trade and port capacity, to achieve efficient operations and better service, it plays a decisive role for the port to have modern technology and management, as well as to implement logistics information.

\section{The Survey of Yingkou Port Logistics Information Technology Implementation}

\section{Overview of Yingkou Port Information Construction}

History and overview of Yingkou Port information development. Since the beginning of 2004, Yingkou Port Group information has entered the application system integration phase, strengthening the integration between each application and, through the company's information 
and effort of each unit, each system has greatly increased its coverage, including the production system, materials management systems, personnel and payroll system, as well as the applications within Hong Kong areas. Application of E-port platform has been in constantly full swing, including OA office systems, material approval systems, management systems, security management systems, and contract approval system.

After the implementation of information, both the organization of production and management have achieved remarkable results. The arrangement of the entire production process, from the human to the machinery distribution, to the latter handling and other steps, all the the cost calculations are to be implemented in computer management. The work efficiency has greatly increased port throughput capability, and significantly increased the number inbound and outbound ships.

\section{Logistics Information Feature of Yingkou Port}

Unity. Yingkou Port logistics information is now EDI-based, but has not yet adopted other information technology. To build a more perfect port logistics electronic platform, it also requires constant technology progress. EDI of Yingkou Port has involved a small part of data transfer instead of more integration into the electronic information system.

Backwardness. Though Yingkou Port is featured by logistics information technology functions, it is still far from perfectness due to the lack of talent. The connection between various functional departments is yet to complete. It is necessary to learn from large international port, and constantly improve the overall aspects.

Low coverage. Among a variety of business of Yingkou Port, the small and medium enterprises account for the majority; the vast majority of small and medium enterprises are not that capable to use information technology for logistics logistics information processing. There are still a lot of information systems still in development and testing phase, also, the coverage is very low. They can not independently complete the tasks even though in some systems that is already in use.

Immediacy. Port is a place in constant progress, and port logistics information needs to catch up with the pace of times to conform to the development needs.

\section{Analysis on Yingkou Port Logistics Information Technology Application}

\section{Swot Analysis}

Yingkou Port logistics information summary. The first few chapters have briefed Yingkou Port logistics information technology application; in general, Yingkou Port has an initial level of electronic logistics information as other giant ports, where EDI is widely used in various logistics processes. However, the participation degree is too low, and it could not comprehensively interpret the functionality of EDI parts; the coverage is also very low. The electronic trading platform under construction has a clean and simple interface, and more comprehensive function, which have well integrated the work of various departments (port logistics as a main) into the Yingkou Port, which becomes a major highlight of logistics information.

Unfavorable factors of Yingkou Port. In addition to the EDI system feature, other international ports are equipped with a complete electronic port platform, which is why Yingkou Port lags behind. Namely: Electronic port affairs platforms, e-commerce platform and e-port. Electronic Port Platform could manage all the affairs within the port, which greatly facilitates the work of internal staff. The construction of e-commerce platform mainly provides external personnel, such as shipping agents, and freight forwarding agents. Those people could understand the ship information, goods information through e-commerce platform, which is indeed a great convenience to users. E-Port has achieved the business between the port, customs and government, in order to facilitate the management and make the process more transparent. 


\section{Problems in Logistics Information of Yingkou Port}

Staff quality of Yingkou Port. Due to the limited geographical location of Yingkou Port, there are not a lot of talents; at the beginning of the implementation of information technology, some relevant technical staff who did not understand the information technology is quite against it; on the other hand, lack of information technology talents lead to the difficulties in optimizing and improving the system.

Effect of organizational business structure. The slow development of logistics information is also decided by the corporate structure, when they can not effectively be combined with each other. In the early period of Yingkou Port Group, the original functions body is designed on the basis of division of specialized labor; in this organization, each man was accustomed to obey superior orders rather than the customer, which made the increase of coordinators during the project. The levels model is no longer suitable to the development of third-generation port, who will no doubt delay logistics operations.

Less comprehensive coverage. Although Yingkou Port has well developed its logistics information, and each system has started to operate and indeed has attained a certain effect, the information is a continuous development and progress process; the coverage degree of the business is yet to be improved. The author believes that there is still a long way to go for Yingkou port to achieve comprehensive port logistics information.

\section{Application Strategy of Logistics Information in Yingkou Port}

To improve the application level of information technology by employee. The biggest obstacle of Yingkou Port logistics information is the awareness of its internal staff, because if researchers people want to implement information technology as a whole, it will inevitably change certain business processes and even the corporate structure, an induce a lot of conflicts of interest and longer implementation process. While at the same time, the staffs are weak in basic knowledge of computers information, some people may be difficult to adapt to the operation of modern information systems, and some may become too dependent on computers. Hence, to improve the level of information technology by employee is very beneficial to the further improvement of Yingkou Port logistics information.

On the whole, logistics information of Yingkou Port should be attached as much importance as a business' development mission. We should promote systems and information technology, organize information technology training, and strengthen information technology heat in all sectors. The system in-use should be accompanied by appropriate personnel training, which is completed by the information company's supervision and guidance of teaching. While the information company's employees should not only master the relevant software and hardware, but also establish the information system in line with Yingkou Port business through learning from major port.

Learn from successful cases. There are many domestic and international giant ports with success, from which researchers can learn from their information technology and apply in the construction of logistics information system for Yingkou Port. In reference to existing model of domestic ports, researchers can also cooperate with third-party experienced or technologically advanced companies, with the purpose of escalating technology to catch up or even exceed other ports in terms of information construction, so as to fully promote the development of port logistics information.

Focus on building "Electronic Port Platform". Electronic Port Platform include OA system, contract management, statistical reporting, material systems management, file system management, fixed asset management, security management, and other functions. Each function can implement multiple functions. Strengthen integration among applications; now researchers are developing appropriate building program for the construction of "Electronic Port Platform", which 
is mainly providing service for the operation and management of our port. It includes the approval of materials and online bidding system, which not only simplifies the process but also reduces costs.

\section{Conclusions}

In the past decade, Yingkou Port has methodically carried out three phases of information technology construction and achieved remarkable results so far; it can be said that the development of Yingkou Port information technology is quite successful. With the global economic integration as well as the business trends represented by customers, competition, and changes, these new changes have proposed new requirements for the development of the port: based on the previous handling foundation, the port should develop into "new era "port" relying on the configuration of information technology.

Yingkou has proposed the goal of "revitalizing the city with harbor, creating large port and building a modern port city". Therefore, application of information technology is crucial for the Yingkou Port. After nearly a decade of difficult course, it finally completed the construction of customized platform for the port itself and has become a port with capabilities over hundred million tons. It has integrated production, engineering, materials, finance and assets, from separate entity of the Group to the joint venture; from the human, financial and material levels it fully reflects logistics information technology of Yingkou Port as a whole. It has learned from the experience of other ports in terms of logistics information technology construction, and once after the solidification of its base, it will also create ERP systems of Port Affairs Group independently towards international port.

\section{References}

[1] Tang Zhiyi. Situation and Characteristics of Ports Information Construction at Home and Abroad. [J] Journal of Central South University of Forestry \& Technology, 2012, (3): 24-29.

[2] Li Xiaogang, Ningbo Logistics Enterprises Development Status and Characteristics of Development. [J] Development and Exploration, 2010, (7): 34-45.

[3] Sun Haitao. Application of EDI technology in the Container Transport Market [J] Network Communications and Security, 2011, (5): 16-29.

[4] Deng Shaoling. Port Logistics Information Platform. [M] Beijing: Beijing People's Communications Press, 2011.5.

[5] Ren Changjiang. Port Logistics [M]. Hangzhou: Zhejiang University Press, 2010.2.

[6] Jiao Xinlong. Study on Port Logistics Performance Evaluation System [J] Chang'an University, 2010, (5): 11-17.

[7] Liu Minghua. Strategies on Strengthening Container Terminal Yard Management [M], Yingkou: Coastal Enterprises and Science \& Technology Press, 2013.6

[8] Cheng Fenglou. Research and Implementation of Port Complex Statistical Management System [J] Journal of Nanjing, 2010, (4): 10-31.

[9] Yang Yanli. Thoughts about Fourth Generation Port Information Technology based on Modern Logistics Development Trend. [J] Port economy, 2012, (3): 26-30.

[10]John Mangan Port-centeric Logistics [J] The International Journal of Logistics 2012(3): 387-401.

[11]Vaidyanathan Jayaraman Anthoy D Ross Anurag Agarwal Role of Information Technology 
and Collaboration in Reverse Logistics Supply Chains [J] International Journal Logistics Research and Applications,2011(5): 82-90. 